\title{
Quadrupole spin polarization as signature of second-order topological superconductors
}

\author{
Kirill Plekhanov $\odot,{ }^{1}$ Niclas Müller $\odot,{ }^{2}$ Yanick Volpez, ${ }^{1}$ Dante M. Kennes, ${ }^{2,3}$ Herbert Schoeller, ${ }^{2}$ \\ Daniel Loss $\odot,{ }^{1}$ and Jelena Klinovaja ${ }^{1}$ \\ ${ }^{1}$ Department of Physics, University of Basel, Klingelbergstrasse 82, CH-4056 Basel, Switzerland \\ ${ }^{2}$ Institut für Theorie der Statistischen Physik, RWTH Aachen, 52056 Aachen, Germany \\ and JARA - Fundamentals of Future Information Technology \\ ${ }^{3}$ Max Planck Institute for the Structure and Dynamics of Matter and Center for Free-Electron Laser Science, 22761 Hamburg, Germany
}

(Received 11 August 2020; accepted 8 December 2020; published 6 January 2021)

\begin{abstract}
We study theoretically second-order topological superconductors characterized by the presence of pairs of zero-energy Majorana corner states. We uncover a quadrupole spin polarization at the system edges that provides a striking signature to identify topological phases, thereby complementing standard approaches based on zero-bias conductance peaks due to Majorana corner states. We consider two different classes of second-order topological superconductors with broken time-reversal symmetry and show that both classes are characterized by a quadrupolar structure of the spin polarization that disappears as the system passes through the topological phase transition. This feature can be accessed experimentally using spin-polarized scanning tunneling microscopes. We study different models hosting second-order topological phases, both analytically and numerically, and using Keldysh techniques we provide numerical simulations of the spin-polarized currents probed by scanning tips.
\end{abstract}

DOI: 10.1103/PhysRevB.103.L041401

Introduction. Over the last two decades, topological insulators (TIs) and superconductors (TSCs) have become a subject of wide interest in condensed matter physics [1-8]. One of the main attractions of such systems is the existence of topologically protected gapless $(d-1)$-dimensional modes which emerge at the boundary of a topologically nontrivial $d$-dimensional bulk-a phenomenon known as bulk-boundary correspondence [9-11]. The topological nature of the boundary modes makes them insensitive to external perturbations and disorder, which is of great importance in the context of quantum computing [12-14]. Recently, the concept of bulk-boundary correspondence has been generalized to a new class of systems, called higher-order topological insulators and superconductors [15-19]. In contrast to conventional topological systems, the $(d-1)$-dimensional boundary of an $n$th order TI/TSC is gapped. Instead, it exhibits protected gapless modes on $(d-n)$-dimensional boundaries. The corresponding gapless modes are called corner states in the case $n=d \geqslant 2$.

Pioneering theoretical works on higher-order TIs have been followed by fast progress from the experimental side [20-30]. Particular attention has been dedicated to theoretical investigations of TSCs that host Majorana corner states (MCSs)-Majorana bound states (MBSs) located at the corners of the system [31-53]. Nevertheless, the experimental realization of such systems remains challenging. Usual protocols to detect MBSs in (higher-order) TSCs are based on a direct state tomography or detection of specific features, such as a zero-bias peak in the differential conductance. However, such probes do not provide a clear way to distinguish between MBSs and other types of bound states of topologically trivial nature [54-68], which hinders unambiguous identification of the topological phases and calls for additional experimental signatures.

In this work, we propose a solution to the problem described above, based on an alternative probe of second-order TSCs (SOTSCs) with broken time-reversal symmetry, which can be implemented with the help of scanning tunneling microscopes (STMs) [69-78]. We consider a subclass of SOTSCs represented by two simple models: the first one supports two MCSs at a single pair of two opposite corners and the second one supports four MCSs-one at every corner of the setup. The topological phase transition in such systems is accompanied by a drastic change in the spin polarization structure at the system edges, which we denote as a quadrupolar structure of the spin polarization. The analytical arguments justifying the emergence of such edge features are confirmed numerically. We then provide results of numerical simulations of the current flowing between a local spin-polarized probe (an STM tip) and the sample, making use of Keldysh techniques. We expect that our approach can also be applied to probe other higher-order topological phases with broken time-reversal symmetry. Importantly, the proposed signatures are stable against weak disorder and do not rely on any other symmetry than the particle-hole symmetry of the superconductor.

SOTSCs with two corner states. To begin with, we consider a class of SOTSCs which supports a single pair of corner states [31-38]. The starting point of our consideration is a simple 2D model for a helical TSC [36], described by the following Hamiltonian in momentum representation:

$$
\begin{aligned}
\mathcal{H}_{0}(\boldsymbol{k})= & 2 t\left[2-\cos \left(k_{x} a\right)-\cos \left(k_{y} a\right)\right] \eta_{z}-\mu_{0} \eta_{z}+\Gamma \eta_{z} \tau_{x} \\
& +\alpha\left[\sin \left(k_{y} a\right) \sigma_{x}-\sin \left(k_{x} a\right) \eta_{z} \sigma_{y}\right] \tau_{z}+\Delta_{\mathrm{sc}} \eta_{y} \sigma_{y} \tau_{z} .
\end{aligned}
$$



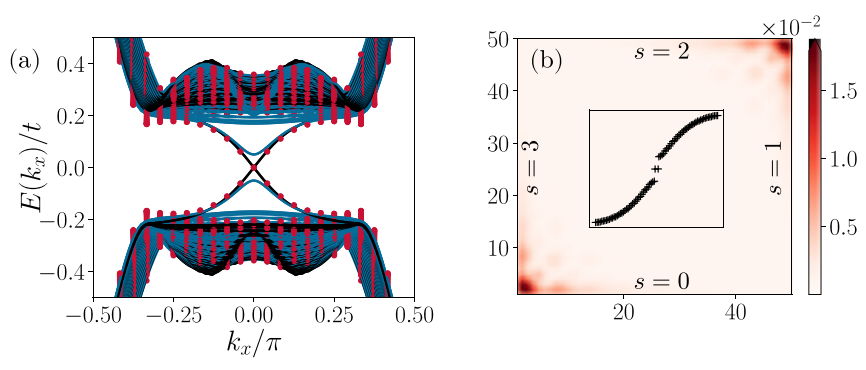

FIG. 1. (a) Spectrum of $\mathcal{H}$ calculated in a geometry with $\mathrm{OBC}$ along the $y$ axis for $\Delta_{\mathrm{z}}=0$ (black lines), $\Delta_{\mathrm{z}}=0.05 t$ and $\theta_{\mathrm{z}}=0$ (blue lines), as well as $\Delta_{\mathrm{z}}=0.05 t$ and $\theta_{\mathrm{z}}=\pi / 2$ (red dots). We see that only the $x$ component of the Zeeman field gaps out the helical edge modes, which are present in the system when $\Delta_{z}=0$. (b) Probability density of the MCSs calculated in a geometry with OBCs along both $x$ and $y$ axis for $\Delta_{z}=0.05 t$ and $\theta_{z}=\pi / 4$. The inset shows the low-energy spectrum. All the simulations are performed in the topological regime of the phase diagram with $\Gamma=2 \Delta_{\mathrm{sc}}=\alpha=$ $0.5 t, \mu_{0}=0$, and $a=1$.

The Pauli matrices $\eta_{j}$ act on the particle-hole space, $\sigma_{j}$ on the spin space, and $\tau_{j}$ on a generic local degree of freedom (e.g., an electron orbital). We work in the Nambu basis $\left(\psi_{\uparrow 1}, \psi_{\downarrow 1}, \psi_{\uparrow 1}^{\dagger}, \psi_{\downarrow 1}^{\dagger}, \psi_{\uparrow \overline{1}}, \psi_{\downarrow \overline{1}}, \psi_{\uparrow \overline{1}}^{\dagger}, \psi_{\downarrow \overline{1}}^{\dagger}\right)$, where $\psi_{\sigma \tau}^{\dagger}$ creates an electron of species $\tau$ and spin $\sigma$. The parameters $\Delta_{\mathrm{sc}}$ and $\mu_{0}$ describe the $s$-wave superconducting pairing amplitude and the chemical potential, respectively, while $t, \alpha$ as well as $\Gamma$ depend on the microscopic details of the system and $a$ is the lattice constant (see Refs. [79-84]). The proposed model is characterized by a topological phase transition at $\Gamma=\Delta_{\mathrm{sc}}$. The region $\Gamma<\Delta_{\text {sc }}$ is trivial, while $\Gamma>\Delta_{\text {sc }}$ corresponds to a helical TSC, supporting a pair of gapless edge modes. The existence of these edge modes is protected by the time-reversal symmetry $T=i \sigma_{y} K$, obeying $T \mathcal{H}_{0}(\boldsymbol{k}) T^{-1}=\mathcal{H}_{0}(-\boldsymbol{k})$, with $K$ being the complex conjugation operator. The topological phase diagram can be checked numerically in a geometry with open boundary condition (OBC) along one fixed direction [see Fig. 1(a)]. The resulting spectrum is independent of the particular choice of the OBC direction as a result of the in-plane rotational symmetry present in the system.

The helical edge modes can be gapped out by applying an external Zeeman field, which breaks time-reversal symmetry. The corresponding contribution to the Hamiltonian can be expressed as

$$
\mathcal{H}_{\mathrm{z}}=\Delta_{\mathrm{z}}\left[\cos \left(\theta_{\mathrm{z}}\right) \eta_{z} \sigma_{x}+\sin \left(\theta_{\mathrm{z}}\right) \sigma_{y}\right],
$$

where $\Delta_{\mathrm{z}}\left(\theta_{\mathrm{z}}\right)$ defines the strength (in-plane orientation) of the Zeeman field. The total Hamiltonian then becomes $\mathcal{H}(\boldsymbol{k})=$ $\mathcal{H}_{0}(\boldsymbol{k})+\mathcal{H}_{\mathrm{z}}$. One can easily show both numerically and analytically that edge modes are gapped out only by the Zeeman field component that is parallel to the edge, see Fig. 1(a). As a result, the rotational symmetry is broken down to the inversion symmetry $I=\eta_{z} \tau_{x}$, which satisfies $I \mathcal{H}(\boldsymbol{k}) I^{-1}=\mathcal{H}(-\boldsymbol{k})$. When the Zeeman field is smaller than the bulk gap of the TSC, $\Delta_{\mathrm{z}} \ll\left|\Gamma-\Delta_{\mathrm{sc}}\right|$, the boundary physics of the system away from the corners is described by a $2 \times 2$ Jackiw-Rebbi Hamiltonian [85,86]

$$
\mathcal{H}_{\text {eff }, s}\left(k_{s}\right)=v_{\mathrm{F}} k_{s} \rho_{z}-m_{s} \rho_{y},
$$

where $\rho_{j}$ act on the space of helical states $\left|\Psi_{0, \pm}^{s}\right\rangle$ belonging to the edge $s \in\{0,1,2,3\}$ [see Fig. 1(b)], $k_{s}$ denotes the momentum parallel to the edge, $v_{\mathrm{F}}$ the Fermi velocity. The strength of the mass term is given by $m_{s}=\Delta_{\mathrm{z}} \cos \left(\theta_{\mathrm{z}}-\theta_{s}\right)$ with $\theta_{s}=$ $s \pi / 2$ (see Ref. [79]). We denote by $\left|\Psi_{0,+}^{s}\right\rangle\left(\left|\Psi_{0,-}^{s}\right\rangle\right.$ ) the states which move clockwise (anticlockwise). Most importantly, as a result of the presence of the inversion symmetry, opposite edges are necessarily described by opposite signs of the mass term $m_{s}$ (i.e., $m_{0}=-m_{2}$ and $m_{1}=-m_{3}$ ). Consequently, in a finite-size geometry where $m_{s}$ is finite on every edge, there exist two corners connecting two edges with opposite signs of $m_{s}$. Such gap inversion corners host zero-energy states identified with MCSs of a SOTSC. Numerical evidence of the existence of MCSs is shown in Fig. 1(b). We note that such a SOTSC phase remains stable against arbitrary types of disorder as long as additional perturbations do not close the surface gap [32-34].

Quadrupolar structure of $\left\langle S_{\perp}\right\rangle$. At low energies, the physics of the setup is dictated by the Zeeman term. In particular, the eigenstates of $\mathcal{H}_{\mathrm{eff}, s}$ at $k_{s}=0$ become spin-polarized and acquire the form

$$
\left|\Psi_{ \pm}^{s}\right\rangle=\left(\left|\Psi_{0,+}^{s}\right\rangle \mp i \operatorname{sgn}\left(m_{s}\right)\left|\Psi_{0,-}^{s}\right\rangle\right) / \sqrt{2}
$$

The states $\left|\Psi_{ \pm}^{s}\right\rangle$ correspond to the eigenvalues $\pm\left|m_{s}\right|$ and have the spin polarization $\left\langle\Psi_{ \pm}^{s}\left|S_{\|}\right| \Psi_{ \pm}^{s}\right\rangle= \pm\left|m_{s}\right| / \Delta_{\mathrm{z}}$, where $S_{\|}$is the spin operator along the direction of the Zeeman field. Similarly, one can calculate the expectation values of the in-plane polarization perpendicular to the applied Zeeman field, associated with the operator $S_{\perp}$. In our case, we use the rotational symmetry of $\mathcal{H}_{0}$ and notice that the gapless states at the edge $s-1$ (where we identify -1 with 3 ) are related to the gapless states at the edge $s$ via a $\pi / 2$-rotation (see Ref. [79]). Hence, the expectation values of $S_{\perp}$ in the basis of states $\left|\Psi_{0, \pm}^{s}\right\rangle$ is exactly equal to the expectation values of $S_{\|}$in the basis $\left|\Psi_{0, \pm}^{s-1}\right\rangle$, resulting in $\left\langle\Psi_{ \pm}^{s}\left|S_{\perp}\right| \Psi_{ \pm}^{s}\right\rangle=$ $\pm \operatorname{sgn}\left(m_{s}\right) m_{s-1} / \Delta_{\mathrm{z}}$. Taking into account that, restricted by the inversion symmetry, the sign of $m_{s}$ changes on every second edge, the sign of the perpendicular component of the spin polarization of low-energy states changes on every edge. We refer to this feature as the quadrupolar structure of the spin polarization.

In Fig. 2, we present the results of a numerical calculation of the spin polarization, based on the Keldysh formalism [73]. We mimic the STM measurement [74-78] by coupling the system to a spin-polarized tip with an amplitude $\kappa$. We then calculate the current $J$ that flows between the system and the tip by summing up the contribution of the states in the energy window $[-V, 0]$ (see Refs. [79,87-89]), where $V$ corresponds to the bias voltage of the STM tip. We consider two directions of the tip polarization: one parallel and one perpendicular to the Zeeman field. As expected from the analytical argument at $k_{s}=0$, we find that in the topological regime, the expectation value of $S_{\|}$is roughly constant and negative, while for $S_{\perp}$ it changes sign on every edge. These features are characteristic for the SOTSCs close to the topological phase transition.

We note that the magnitude of spin polarization may depend on the model-dependent properties of the states $\left|\Psi_{0, \pm}^{s}\right\rangle$ and the symmetries of $\mathcal{H}_{0}$ (see Refs. [79,90-95]). Nevertheless, the quadrupolar structure associated with the sign of the 

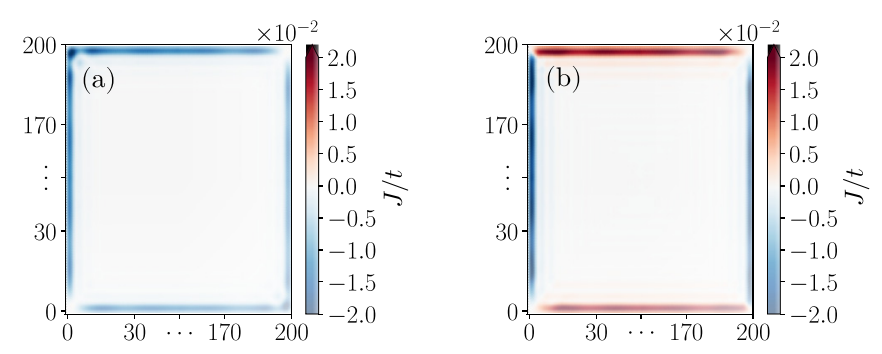

FIG. 2. Numerical calculations of the current $J$ through the spinpolarized STM tip for a system described by $\mathcal{H}$ given below Eq. (2). The polarization of the STM is chosen to be in-plane (a) parallel to the Zeeman field and (b) perpendicular to the Zeeman field. The outof-plane component is trivially zero. We see that while the parallel component of the polarization is roughly constant and negative along the entire boundary, the perpendicular component has a quadrupolar structure, i.e., changes sign on every edge. The parameters of the simulation are $\Gamma=1.75 \Delta_{\mathrm{sc}}=0.44 t, \Delta_{\mathrm{z}}=0.05 t, \kappa=2.5 \cdot 10^{-4} t$, and the contribution to the current is summed over $V$ from 0 to $\Delta_{\mathrm{z}}$.

spin polarization at different edges depends only on the sign of $m_{s}$, which are topologically protected quantities. Hence, observing a quadrupolar structure of the spin-polarization provides a prominent and unique signature of the system topology, universal for this class of SOTSCs. Moreover, the effective edge Hamiltonian $\mathcal{H}_{\text {eff, } s}$ as well as the argument justifying the emergence of MCSs are also generally valid for inversion-symmetric SOTSCs [31-34].

SOTSCs with four corner states. Next, we generalize our findings to a different class of SOTSCs and consider a SOTSC hosting a quadruplet of MCSs [39-48]. The basic ingredient of our construction here is a minimalist version of a $2 \mathrm{D}$ TI [3] proximity coupled to an $s$-wave superconductor with amplitude $\Delta_{\mathrm{sc}}$, introduced in Ref. [47]. The corresponding Hamiltonian reads

$$
\begin{aligned}
\mathcal{H}_{0}^{\prime}(\boldsymbol{k})= & \left\{\Gamma-2 t_{x}\left[1-\cos \left(k_{x} a\right)\right]-2 t_{y}\left[1-\cos \left(k_{y} a\right)\right]\right\} \eta_{z} \tau_{z} \\
& -\mu_{0} \eta_{z}+\left[\alpha_{x} \sin \left(k_{x} a\right) \sigma_{z} \tau_{x}-\alpha_{y} \sin \left(k_{y} a\right) \eta_{z} \tau_{y}\right] \\
& +\Delta_{\mathrm{sc}} \eta_{y} \sigma_{y}
\end{aligned}
$$

where Pauli matrices $\eta_{j}, \sigma_{j}$, and $\tau_{j}$ play exactly the same role as in Eq. (1). The parameters $t_{x}, t_{y}, \alpha_{x}, \alpha_{y}$, and $\Gamma$ depend on the microscopic details of the system (see Ref. [79]). For $\Delta_{\mathrm{sc}}=0$ the system is characterized by a topological phase transition as a function of the parameter $\Gamma$. The closing of the gap occurs at $\Gamma=0$, independently of the value of $\alpha_{x}$ and $\alpha_{y}$. The region $\Gamma<0$ is topologically trivial, while $\Gamma>0$ is identified with a TI phase that supports gapless helical edge modes protected by the time-reversal symmetry $T=i \sigma_{y} K$. Again, we verify the presence of such edge modes by analyzing the model numerically in a geometry with $\mathrm{OBC}$ along one particular axis. The result of the calculation is shown in Fig. 3(a).

At nonzero $\Delta_{\mathrm{sc}}$, the helical edge modes are gapped out. However, such a process acts identically on all the edges, transforming the system into a trivial superconductor. In order to achieve richer physics, one can apply the in-plane Zeeman field, described by the Hamiltonian term

$$
\mathcal{H}_{\mathrm{z}}^{\prime}=\Delta_{\mathrm{z}} \eta_{z} \sigma_{x},
$$
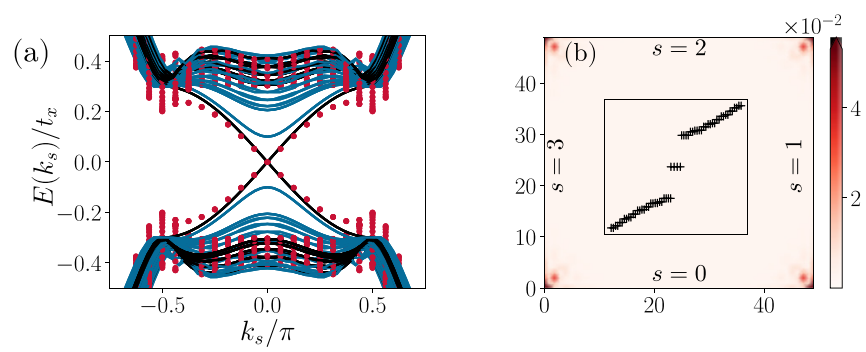

FIG. 3. (a) Spectrum of $\mathcal{H}^{\prime}$ for $\Delta_{\mathrm{sc}}=0$. When $\Delta_{\mathrm{z}}=0$ (black lines), the system hosts a pair of gapless helical edge modes. A finite value of $\Delta_{\mathrm{z}}=0.1 t_{x}$ gaps out the edge modes in a geometry with OBC along the $y$ axis (blue lines, $k_{s} \equiv k_{x}$ ) but leaves the edge modes gapless in a geometry with OBC along the $x$ axis (red dots, $k_{s} \equiv$ $k_{y}$ ). (b) Probability density of the MCSs calculated in a geometry with OBCs along both $x$ and $y$ axes in a topological regime of the phase diagram with $\Delta_{\mathrm{z}}=2 \Delta_{\mathrm{sc}}=0.1 t_{x}$. The inset shows the lowenergy spectrum. The remaining parameters are $\Gamma=t_{y}=t_{x}, \alpha_{x}=$ $\alpha_{y}=0.3 t_{x}, \mu_{0}=0$, and $a=1$.

such that the total Hamiltonian becomes $\mathcal{H}^{\prime}(\boldsymbol{k})=\mathcal{H}_{0}^{\prime}(\boldsymbol{k})+$ $\mathcal{H}_{\mathrm{z}}^{\prime}$. The resulting system is invariant under the inversion symmetry $I=\tau_{z}$. We also note that the exact orientation of the Zeeman field in the $x y$ plane is not important, since the spectrum is invariant under an arbitrary rotation around the spin quantization axis. Interestingly, the effect of the in-plane Zeeman field alone differs strongly depending on the edge considered. This can be seen by considering two special lines in the momentum space, corresponding to vanishing $k_{x}$ or $k_{y}$, which can be used to describe the physics of the system in a geometry with OBC along the $y$ or $x$ axis, respectively (see Ref. [79]). For $k_{y}=0$, the Zeeman term leaves the edge modes gapless, while for $k_{x}=0$ it leads to an opening of the gap of the size $\Delta_{\mathrm{z}}$. As a consequence, the modes propagating along $x$ edges of the system are gapped, while the ones along the $y$ edges remain gapless. The numerical verification of this feature is shown in Fig. 3(a).

By taking into account the effect of both the superconducting and Zeeman terms, one can construct the low-energy effective $4 \times 4$ edge Hamiltonian, which reads

$$
\mathcal{H}_{\text {eff }, s}^{\prime}\left(k_{s}\right)=v_{\mathrm{F}} k_{s} \rho_{z}+m_{s} \eta_{z} \rho_{x}+\Delta_{\mathrm{sc}} \eta_{y} \rho_{y} .
$$

Here, we use the same convention as in Eq. (3) and denote by $\rho_{j}$ the matrices acting on the states $\left|\Psi_{0, \pm}^{\prime s}\right\rangle$, associated with the right- and left-moving modes living on the edge $s \in\{0,1,2,3\}$ [see Fig. 3(b)]. The matrices $\eta_{j}$ act in the particle-hole space and $m_{s}$ denotes the mass originating from the Zeeman term. The mass vanishes on two $y$ edges: $m_{1}=$ $m_{3}=0$, while $\left|m_{0}\right|=\left|m_{2}\right|=\Delta_{z}$ on two $x$ edges. Moreover, the effective description of every edge is identical to the low-energy physics of a topological nanowire [96-100], characterized by the topological phase transition at a critical point $\Delta_{\mathrm{z}}=\Delta_{\mathrm{sc}}$. Hence, if the Zeeman field is strong enough such that $\Delta_{\mathrm{z}}>\Delta_{\mathrm{sc}}$, the two $x$ edges of the system correspond to two wires in the topological regime, while the two $y$ edges - to two trivial nanowires. As a result, four corners of the system host four zero energy states, identified with MCSs. The regime $\Delta_{\mathrm{z}}<\Delta_{\mathrm{sc}}$ is the topologically trivial phase. Similarly, the corner states disappear when the system moves 

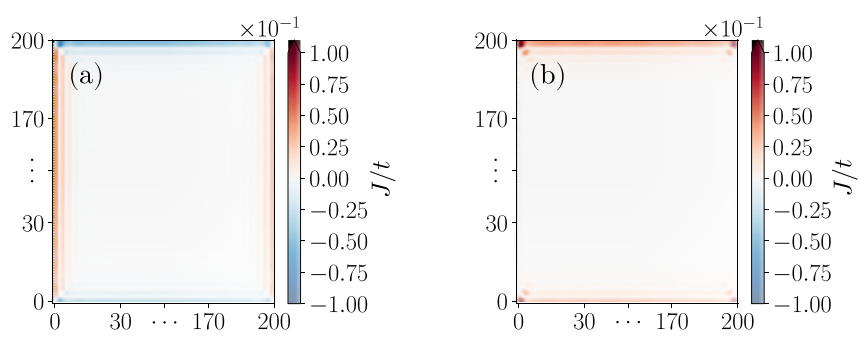

FIG. 4. Numerical calculation of the current $J$ through the spinpolarized STM tip for a system described by $\mathcal{H}^{\prime}$ given below Eq. (6). The polarization of the STM is chosen to be parallel to the Zeeman field. (a) In the topological phase with $\Delta_{\mathrm{z}}=1.75 \Delta_{\mathrm{sc}}$, the polarization changes sign on every edge, resulting in a quadrupolar structure similar to the one of Fig. 2. (b) In the topologically trivial phase with $\Delta_{\mathrm{z}}=0.25 \Delta_{\mathrm{sc}}$, the polarization has the same sign along the entire system boundary. The signal is weaker along the $y$ edges because less edge states contribute in the energy window. The remaining parameters of the simulations are $\Gamma=t_{y}=t_{x}, \alpha_{y}=\alpha_{x}=0.3 t_{x} \Delta_{\mathrm{sc}}=0.1 t_{x}$, and $\kappa=5 \times 10^{-4} t_{x}$. The current contributions are summed up over $V=\Delta_{\text {sc }}$.

into the trivial region of the phase diagram with $\Gamma<0$. We also note that the topological description of the present model does not rely on the presence of the inversion symmetry $I$. Instead, the existence of four MCSs is ensured by a particular spatial structure of the gapping processes $m_{s}$ and $\Delta_{\text {sc }}$ generic to this class of SOTSCs, namely, by the fact that one pair of opposite edges has $\left|m_{s}\right|<\Delta_{\text {sc }}$, while the other one has $\left|m_{s}\right|>\Delta_{\mathrm{sc}}$.

Quadrupolar structure of $\left\langle S_{\|}\right\rangle$. We show that, again, a spinpolarized STM [73-78] can be used to probe the topological phase diagram of $\mathcal{H}_{\mathrm{eff}, s}^{\prime}$. To show this, we consider the edges $s=0,2$ and focus on the physics at the Dirac point $k_{s}=0$, where the phase transition occurs. We denote by $\left|\Psi_{ \pm}^{\prime s}\right\rangle$ two eigenstates of $\mathcal{H}_{\text {eff, } s}^{\prime}$ associated with two lowest magnitude eigenvalues $\pm\left|m_{s}-\Delta_{\mathrm{sc}}\right|$. The states $\left|\Psi_{ \pm}^{\prime s}\right\rangle$ are eigenstates of the Zeeman term and are characterized by the polarization $\left\langle\Psi_{ \pm}^{\prime s}\left|S_{\|}\right| \Psi_{ \pm}^{\prime s}\right\rangle= \pm 1$ for $\Delta_{\mathrm{z}}>\Delta_{\text {sc }}$. The polarization of these two states remains constant up to the point $\Delta_{\mathrm{z}}=\Delta_{\mathrm{sc}}$, at which the gap is closed at the edges and the topological phase transition occurs. Decreasing the Zeeman term further, the spin polarization of these two states flip, acquiring a new value $\left\langle\Psi_{ \pm}^{\prime s}\left|S_{\|}\right| \Psi_{ \pm}^{\prime s}\right\rangle=\mp 1$. At the same time, no gap closing occurs at the edges $s=1,3$. As a consequence, the expectation value of $S_{\|}$grows smoothly as a function of the ratio ratio $\Delta_{\mathrm{z}} / \Delta_{\mathrm{sc}}$ without flipping its sign. Hence, the parallel component of the spin polarization changes sign only on two $m_{s} \neq 0$ edges of the system as one goes through the topological phase transition and it acquires the quadrupolar structure only in the topological phase but not in the trivial one (see Ref. [79]). The expectation values of perpendicular components of the spin polarization are zero. This feature allows one to unambiguously identify the topological phase transition occurring in such a system.

These analytical predictions in low-energy approximation are confirmed by a numerical study of $\mathcal{H}^{\prime}$, presented in Fig. 4 , where we calculate the current through the spin-polarized STM tip (see Ref. [79]). When $\Gamma>0$, we find that the expectation values of $S_{\|}$are always positive along the two $y$ edges and are positive (negative) when $\Delta_{\mathrm{z}}<\Delta_{\mathrm{sc}}\left(\Delta_{\mathrm{z}}>\Delta_{\mathrm{sc}}\right)$ along the two $x$ edges. When $\Gamma<0$, the description in terms of $\mathcal{H}_{\text {eff, } s}$ breaks down and the bulk signal dominates over the edge signal. As expected, the perpendicular components of the spin polarization is found to be negligibly small everywhere except at the system corners. We have also checked that the proposed feature is stable against weak disorder.

Conclusions. In this work, we analyzed the spin polarization of two-dimensional topological superconductors as a signature indicating topological phases. In particular, we showed that a spin-polarized STM can be used to determine the topological phase diagram in two types of second-order topological superconductors (SOTSCs) with broken timereversal symmetry. In SOTSCs, which host a pair of corner states, the distinguishing feature of the topological phase is the quadrupolar structure of the spin polarization perpendicular to the Zeeman field. Similarly, in SOTSCs with two pairs of corner states, the spin polarization parallel to the Zeeman field acquires such a quadrupolar structure only in the topological phase. This probe can be used in conjunction with the usual experimental protocols, such as the state tomography and the measurement of the differential conductance, to verify the topological nature of bound states and serves as an additional independent signature of the topological phase transition.

Acknowledgments. We gratefully acknowledge many useful discussions with Katharina Laubscher, Flavio Ronetti, and Ferdinand Schulz. This work was supported by the Swiss National Science Foundation, NCCR QSIT, and the Georg H. Endress foundation as well as the Deutsche Forschungsgemeinschaft via RTG 1995 and by the Deutsche Forschungsgemeinschaft (DFG, German Research Foundation) under Germany's Excellence Strategy - Cluster of Excellence Matter and Light for Quantum Computing (ML4Q) EXC 2004/1390534769. This project received funding from the European Union's Horizon 2020 research and innovation program (ERC Starting Grant, grant agreement No 757725). We acknowledge support from the Max Planck-New York City Center for Non-Equilibrium Quantum Phenomena. Simulations were performed with computing resources granted by RWTH Aachen University under projects rwth0498, rwth0563, rwth0564 and rwth0593.
[1] C. L. Kane and E. J. Mele, Phys. Rev. Lett. 95, 226801 (2005).

[2] C. L. Kane and E. J. Mele, Phys. Rev. Lett. 95, 146802 (2005).

[3] B. A. Bernevig, T. L. Hughes, and S. Zhang, Science 314, 1757 (2006).

[4] M. Z. Hasan and C. L. Kane, Rev. Mod. Phys. 82, 3045 (2010).
[5] X.-L. Qi and S.-C. Zhang, Rev. Mod. Phys. 83, 1057 (2011).

[6] M. Sato and Y. Ando, Rep. Prog. Phys. 80, 076501 (2017).

[7] J. Wang and S.-C. Zhang, Nat. Mater. 16, 1062 (2017).

[8] X.-G. Wen, Rev. Mod. Phys. 89, 041004 (2017).

[9] R. B. Laughlin, Phys. Rev. B 23, 5632(R) (1981). 
[10] C. G. Callan Jr. and J. A. Harvey, Nucl. Phys. B 250, 427 (1985).

[11] T. L. Hughes, R. G. Leigh, and O. Parrikar, Phys. Rev. D 88, 025040 (2013).

[12] M. H. Freedman, A. Kitaev, M. J. Larsen, and Z. Wang, Bull. Amer. Math. Soc. 40, 31 (2003).

[13] A. Kitaev, Ann. Phys. 303, 2 (2003).

[14] A. Stern and N. H. Lindner, Science 339, 1179 (2013).

[15] R.-J. Slager, L. Rademaker, J. Zaanen, and L. Balents, Phys. Rev. B 92, 085126 (2015).

[16] W. A. Benalcazar, B. A. Bernevig, and T. L. Hughes, Science 357, 61 (2017).

[17] W. A. Benalcazar, B. A. Bernevig, and T. L. Hughes, Phys. Rev. B 96, 245115 (2017).

[18] Z. Song, Z. Fang, and C. Fang, Phys. Rev. Lett. 119, 246402 (2017).

[19] J. Langbehn, Y. Peng, L. Trifunovic, F. von Oppen, and P. W. Brouwer, Phys. Rev. Lett. 119, 246401 (2017).

[20] C. W. Peterson, W. A. Benalcazar, T. L. Hughes, and G. Bahl, Nature 555, 346 (2018).

[21] S. Imhof, C. Berger, F. Bayer, J. Brehm, L. W. Molenkamp, T. Kiessling, F. Schindler, C. H. Lee, M. Greiter, T. Neupert, and R. Thomale, Nat. Phys. 14, 925 (2018).

[22] S. Mittal, V. V. Orre, G. Zhu, M. A. Gorlach, A. Poddubny, and M. Hafezi, Nat. Photon. 13, 692 (2019).

[23] X.-D. Chen, W.-M. Deng, F.-L. Shi, F.-L. Zhao, M. Chen, and J.-W. Dong, Phys. Rev. Lett. 122, 233902 (2019).

[24] A. E. Hassan, F. K. Kunst, A. Moritz, G. Andler, E. J. Bergholtz, and M. Bourennane, Nat. Photon. 13, 697 (2019).

[25] M. Serra-Garcia, V. Peri, R. Süsstrunk, O. R. Bilal, T. Larsen, L. G. Villanueva, and S. D. Huber, Nature 555, 342 (2018).

[26] H. Xue, Y. Yang, F. Gao, Y. Chong, and B. Zhang, Nat. Mater. 18, 108 (2019).

[27] F. Schindler, A. M. Cook, M. G. Vergniory, Z. Wang, S. S. P. Parkin, B. A. Bernevig, and T. Neupert, Sci. Adv. 4, eaat0346 (2018).

[28] F. Schindler, Z. Wang, M. G. Vergniory, A. M. Cook, A. Murani, S. Sengupta, A. Y. Kasumov, R. Deblock, S. Jeon, I. Drozdov, H. Bouchiat, S. Guëron, A. Yazdani, B. A. Bernevig, and T. Neupert, Nat. Phys. 14, 918 (2018).

[29] X. Ni, M. Weiner, A. Alú, and A. B. Khanikaev, Nat. Mater. 18, 113 (2019).

[30] X. Zhang, B.-Y. Xie, H.-F. Wang, X. Xu, Y. Tian, J.-H. Jiang, M.-H. Lu, and Y.-F. Chen, Nat. Commun. 10, 5331 (2019).

[31] K. Shiozaki and M. Sato, Phys. Rev. B 90, 165114 (2014).

[32] M. Geier, L. Trifunovic, M. Hoskam, and P. W. Brouwer, Phys. Rev. B 97, 205135 (2018).

[33] E. Khalaf, Phys. Rev. B 97, 205136 (2018).

[34] L. Trifunovic and P. W. Brouwer, Physica Status Solidi (b) (2020), doi: 10.1002/pssb.202000090.

[35] X. Zhu, Phys. Rev. B 97, 205134 (2018).

[36] Y. Volpez, D. Loss, and J. Klinovaja, Phys. Rev. Lett. 122, 126402 (2019).

[37] K. Laubscher, D. Loss, and J. Klinovaja, Phys. Rev. Research 1, 032017 (2019).

[38] J. Ahn and B.-J. Yang, Phys. Rev. Research 2, 012060(R) (2020).

[39] Y. Wang, M. Lin, and T. L. Hughes, Phys. Rev. B 98, 165144 (2018).

[40] T. Liu, J. J. He, and F. Nori, Phys. Rev. B 98, 245413 (2018).
[41] S. Franca, D. V. Efremov, and I. C. Fulga, Phys. Rev. B 100, 075415 (2019).

[42] Z. Yan, Phys. Rev. B 100, 205406 (2019).

[43] R.-X. Zhang, W. S. Cole, X. Wu, and S. Das Sarma, Phys. Rev. Lett. 123, 167001 (2019).

[44] X. Zhu, Phys. Rev. Lett. 122, 236401 (2019).

[45] K. Laubscher, D. Loss, and J. Klinovaja, Phys. Rev. Research 2, 013330 (2020).

[46] X. Wu, X. Liu, R. Thomale, and C.-X. Liu, arXiv:1905.10648.

[47] Y.-J. Wu, J. Hou, Y.-M. Li, X.-W. Luo, and C. Zhang, Phys. Rev. Lett. 124, 227001 (2020).

[48] S.-B. Zhang, A. Calzona, and B. Trauzettel, Phys. Rev. B 102, 100503 (2020).

[49] Z. Yan, F. Song, and Z. Wang, Phys. Rev. Lett. 121, 096803 (2018).

[50] Q. Wang, C.-C. Liu, Y.-M. Lu, and F. Zhang, Phys. Rev. Lett. 121, 186801 (2018).

[51] C.-H. Hsu, P. Stano, J. Klinovaja, and D. Loss, Phys. Rev. Lett. 121, 196801 (2018).

[52] L. Chen, B. Liu, G. Xu, and X. Liu, arXiv:1909.10402.

[53] Y.-T. Hsu, W. S. Cole, R.-X. Zhang, and J. D. Sau, Phys. Rev. Lett. 125, 097001 (2020).

[54] R. Zitko, J. S. Lim, R. López, and R. Aguado, Phys. Rev. B 91, 045441 (2015).

[55] E. Prada, P. San-Jose, M. W. A. de Moor, A. Geresdi, E. J. H. Lee, J. Klinovaja, D. Loss, J. Nygard, R. Aguado, and L. P. Kouwenhoven, Nat. Rev. Phys. 2, 575 (2020).

[56] C. Reeg, O. Dmytruk, D. Chevallier, D. Loss, and J. Klinovaja, Phys. Rev. B 98, 245407 (2018).

[57] A. Ptok, A. Kobialka, and T. Domanski, Phys. Rev. B 96, 195430 (2017).

[58] F. Setiawan, C.-X. Liu, J. D. Sau, and S. Das Sarma, Phys. Rev. B 96, 184520 (2017).

[59] C. Moore, T. D. Stanescu, and S. Tewari, Phys. Rev. B 97, 165302 (2018).

[60] A. Vuik, B. Nijholt, A. R. Akhmerov, and M. Wimmer, SciPost Phys. 7, 061 (2019).

[61] J. Avila, F. Penaranda, E. Prada, P. San-Jose, and R. Aguado, Commun. Phys. 2, 133 (2019).

[62] E. B. Hansen, J. Danon, and K. Flensberg, Phys. Rev. B 97, 041411(R) (2018).

[63] F. Penaranda, R. Aguado, P. San-Jose, and E. Prada, Phys. Rev. B 98, 235406 (2018).

[64] G. Kells, D. Meidan, and P. W. Brouwer, Phys. Rev. B 86, 100503(R) (2012).

[65] C. Fleckenstein, F. Dominguez, N. Traverso Ziani, and B. Trauzettel, Phys. Rev. B 97, 155425 (2018).

[66] C. Jünger, R. Delagrange, D. Chevallier, S. Lehmann, K. A. Dick, C. Thelander, J. Klinovaja, D. Loss, A. Baumgartner, and C. Schönenberger, Phys. Rev. Lett. 125, 017701 (2020).

[67] D. J. Alspaugh, D. E. Sheehy, M. O. Goerbig, and P. Simon, Phys. Rev. Research 2, 023146 (2020).

[68] S.-B. Zhang and B. Trauzettel, Phys. Rev. Research 2, 012018 (2020).

[69] P. Szumniak, D. Chevallier, D. Loss, and J. Klinovaja, Phys. Rev. B 96, 041401(R) (2017).

[70] M. Serina, D. Loss, and J. Klinovaja, Phys. Rev. B 98, 035419 (2018).

[71] M. Thakurathi, D. Chevallier, D. Loss, and J. Klinovaja, Phys. Rev. Research 2, 023197 (2020). 
[72] M. Mashkoori, S. Pradhan, K. Bjornson, J. Fransson, and A. M. Black-Schaffer, Phys. Rev. B 102, 104501 (2020).

[73] N. Müller, D. M. Kennes, J. Klinovaja, D. Loss, and H. Schoeller, Phys. Rev. B 101, 155417 (2020).

[74] C. Jozwiak, J. A. Sobota, K. Gotlieb, A. F. Kemper, C. R. Rotundu, R. J. Birgeneau, Z. Hussain, D.-H. Lee, Z.-X. Shen, and A. Lanzara, Nat. Commun. 7, 13143 (2016).

[75] S. Jeon, Y. Xie, J. Li, Z. Wang, B. A. Bernevig, and A. Yazdani, Science 358, 772 (2017).

[76] B. Jäck, Y. Xie, J. Li, S. Jeon, B. A. Bernevig, and A. Yazdani, Science 364, 1255 (2019).

[77] H. Kim, A. Palacio-Morales, T. Posske, L. Rózsa, K. Palotás, L. Szunyogh, M. Thorwart, and R. Wiesendanger, Sci. Adv. 4, eaar5251 (2018).

[78] M. Ruby, B. W. Heinrich, Y. Peng, F. von Oppen, and K. J. Franke, Nano Lett. 17, 4473 (2017).

[79] See Supplemental Material at http://link.aps.org/supplemental/ 10.1103/PhysRevB.103.L041401 for (1) microscopic details of the models; (2) details on the numerical methods; (3) details on the analytical calculations of the edge states and the spin polarization; (4) a study of the quadrupolar moment across the phase transition; and (5) the stability of the quadrupolar features against external perturbations.

[80] M. König, S. Wiedmann, C. Brüne, A. Roth, H. Buhmann, L. W. Molenkamp, X. Qi, and S. Zhang, Science 318, 766 (2007).

[81] A. Roth, C. Brüne, H. Buhmann, L. W. Molenkamp, J. Maciejko, X. L. Qi, and S. C. Zhang, Science 325, 294 (2009).

[82] C. Liu, T. L. Hughes, X. L. Qi, K. Wang, and S. C. Zhang, Phys. Rev. Lett. 100, 236601 (2008).
[83] C. L. Kane, R. Mukhopadhyay, and T. C. Lubensky, Phys. Rev. Lett. 88, 036401 (2002).

[84] J. C. Y. Teo and C. L. Kane, Phys. Rev. B 89, 085101 (2014).

[85] R. Jackiw and C. Rebbi, Phys. Rev. D 13, 3398 (1976).

[86] R. Jackiw and J. Schrieffer, Nucl. Phys. B 190, 253 (1981).

[87] D. Chevallier, P. Szumniak, S. Hoffman, D. Loss, and J. Klinovaja, Phys. Rev. B 97, 045404 (2018).

[88] D. Chevallier and J. Klinovaja, Phys. Rev. B 94, 035417 (2016).

[89] M. M. Maska and T. Domanski, Sci. Rep. 7, 16193 (2017).

[90] J. Klinovaja and D. Loss, Phys. Rev. B 86, 085408 (2012).

[91] D. Sticlet, C. Bena, and P. Simon, Phys. Rev. Lett. 108, 096802 (2012).

[92] S. B. Zhang, Y. Y. Zhang, and S. Q. Shen, Phys. Rev. B 90, 115305 (2014).

[93] R. Skolasinski, D. I. Pikulin, J. Alicea, and M. Wimmer, Phys. Rev. B 98, 201404(R) (2018).

[94] C. A. Li, S. B. Zhang, and S. Q. Shen, Phys. Rev. B 97, 045420 (2018).

[95] F. Schulz, K. Plekhanov, D. Loss, and J. Klinovaja, Phys. Rev. Research 2, 033215 (2020).

[96] J. Nilsson, A. R. Akhmerov, and C. W. J. Beenakker, Phys. Rev. Lett. 101, 120403 (2008).

[97] L. Fu and C. L. Kane, Phys. Rev. B 79, 161408(R) (2009).

[98] R. M. Lutchyn, J. D. Sau, and S. Das Sarma, Phys. Rev. Lett. 105, 077001 (2010).

[99] Y. Oreg, G. Refael, and F. von Oppen, Phys. Rev. Lett. 105, 177002 (2010).

[100] J. Alicea, Phys. Rev. B 81, 125318 (2010). 